Rev. Adm. Saúde - Vol. 18, № 73, out. - dez. 2018

http://dx.doi.org/10.23973/ras.73.142

ARTIGO DE REVISÃO

\title{
Atuação do enfermeiro nas estratégias para resolução de conflitos
}

Nurse's performance with conflict resolution strategies

\section{Ana Caroline Duarte Souza ${ }^{1}$, Fabiana Souza Silva², Juliana Silva Espindola ${ }^{3}$, Natalia Lima Moreira ${ }^{4}$, Patricia Bover Draganov ${ }^{5}$}

\author{
1, 2, 3, 4. Graduanda de enfermagem. Universidade Anhembi Morumbi, São Paulo SP \\ 5. Enfermeira. Docente da Universidade Anhembi Morumbi, São Paulo SP
}

\section{RESUMO}

Introdução: Saber lidar com situações de conflitos e como eles tem influência na vida das pessoas é fundamental para o enfermeiro, que no papel de líder gerencia sua equipe de enfermagem nas atribuições a assistência. Objetivo: Descrever estratégias resolutivas de negociação de conflitos na enfermagem. Metodologia: Este estudo trata-se de uma revisão bibliográfica da literatura. Como critério de seleção dos artigos, foram utilizados aqueles publicados nos últimos 10 anos (período de 2008 a 2018) e indexados nas bases de BDENF e LILACS. Resultados: Foram selecionados 14 artigos e 1 livro para o desenvolvimento deste estudo. De acordo com a pesquisa realizada, foram criadas as categorias: descrever conflitos e os aspectos na área da saúde e enfermagem; estratégias de negociação na enfermagem; estratégias resolutivas - infográfico. Considerações finais: Através deste estudo exploramos as estratégias mais utilizadas pelo enfermeiro para solução de conflitos, destacando temas de supra importância para o entendimento do assunto. Ter um bom relacionamento e uma comunicação eficaz com a sua equipe, além de gerar confiança, os mesmos poderão se sentir mais à vontade para trazer um conflito à gerência, ganhando a oportunidade de crescer como um grande líder; conquistando um ambiente de trabalho mais equilibrado e agradável para os mesmos. 
Palavras-chave: Estratégia; Conflito; Negociação; Gestão Hospitalar; Enfermagem

\begin{abstract}
Know how to deal with conflict situations and how they have influence on the lives of people is fundamental for the nurse, who in the role of leader manages his nursing team in assignments to assistance. Goal: To describe resolution strategies of negotiation of conflicts in Nursing. Methods: This study is a literature review. As a criterion for the selection of articles, were used those published in the last 10 years (2008 to 2018) and indexed in databases BDENF and LILACS. Results: Were selected 14 articles and 1 book for the development of this study. According to the survey, were created the categories: Describe conflicts and the aspects in the area of health and nursing; negotiation strategies in Nursing; Resolution strategies - Infographic. Final
\end{abstract} Considerations: This study explored the strategies used by nurses to resolve conflicts, highlighting important themes for understanding the subject. With a good relationship and effective communication with your team, in addition to generating trust, they may feel more comfortable conducting conflict management, gaining the opportunity to grow as a great leader; a more balanced and pleasant work environment for them.

Keywords: Strategy; Conflict; Negotiation; Hospital Management; Nursing.

\title{
INTRODUÇÃO
}

A enfermagem envolve o trabalho conjunto a todas as outras áreas dentro de estabelecimentos assistenciais de saúde na busca de assistência de qualidade pautadas em bases cientificas. ${ }^{1}$

O enfermeiro lidera equipe de enfermagem nas atribuições a assistência e saber lidar com situações conflituosas e como elas influenciam na vida das pessoas é fundamental para o enfermeiro. O conflito é esperado como consequência das reações entre opiniões, valores e conceitos diferentes e por isso a probabilidade de surgir um atrito por qualquer que seja o motivo é enorme. Cabe ao enfermeiro solucionar, minimizar as diferenças de percepção entre os envolvidos, gerenciar a diversidade, saber ouvir e saber se expressar, tratar as pessoas com respeito e compreendendo que as diferenças podem conduzir o crescimento pessoal e profissional. ${ }^{1}$

O conflito é definido como o desacordo interno ou externo resultante de diferenças de ideias e valores entre duas ou mais pessoas. Nesse contexto, os conflitos podem advir de relacionamentos com pessoas que possuem crenças, 
formação, metas, valores profissionais divergentes e expectativas profissionais mal definidas dentro do ambiente institucional. ${ }^{2}$

São cinco os estágios que compreendem o processo do conflito, é importante que essas fases sejam reconhecidas para que as situações possam ser resolvidas de forma rápida e da melhor maneira possível, caso o contrário pode haver minimamente, queda de produtividade, da qualidade nos serviços prestados e desmotivação prejudicando todos os envolvidos. ${ }^{3}$

A negociação é um processo multifacetário que se utiliza de ferramentas e atitudes tais como boa comunicação, flexibilidade e liderança para concentrar interesses em uma mesma condição de igualdade, sabendo resolver os problemas sem gerar ansiedade e desconforto. ${ }^{1}$

Saber negociar é imprescindível na resolução de um desacordo e é necessário ser flexível, firme e amistoso, franco e persuasivo. Negociar não é atacar ou ceder, é conversar sobre conciliação e fazer acordos eficazes em que ambos ganhem. ${ }^{1}$

O processo de negociação consiste nas etapas de preparação, comunicação assertiva, motivação, controle e avaliação. ${ }^{4}$

Estratégias são ações que ao serem executadas melhoram o posicionamento da empresa, favorecendo o ambiente, a equipe e os clientes. ${ }^{4}$

A pergunta problema deste estudo é: quais as estratégias usadas pelo enfermeiro na resolução de conflitos?

A hipótese é que são utilizadas técnicas de negociações que possam ajudar a solucionar tais conflitos, buscando um ambiente de trabalho harmônico.

Esse estudo se justifica, pois, a resolução de conflitos pelo enfermeiro com uso de estratégias resolutivas contribui para excelência da qualidade prestada e para a satisfação profissional.

\section{OBJETIVO GERAL}

O objetivo desse estudo foi descrever estratégias resolutivas de negociação de conflitos na enfermagem.

Para atender ao objetivo geral. Tende-se como objetivos específicos:

1. Descrever conflitos e os aspectos na área da saúde e enfermagem;

2. Estratégias de negociação na enfermagem;

3. Estratégias resolutivas - Infográfico. 


\section{MÉTODO}

O método científico utilizado neste estudo foi o de pesquisa de revisão bibliográfica, exposição teórica sobre o assunto, destacando pesquisas já publicadas e os principais trabalhos científicos existentes na área, atendendo a pesquisa de título atuação do enfermeiro com estratégia para resolução de conflito.

A ideia de uma revisão bibliográfica é enunciar alguns dos 'interlocutores' com os quais você travará o seu diálogo historiográfico e científico. Estes interlocutores constituirão parte da riqueza de seu trabalho, e não convém negligenciá-los. O que se pede na revisão bibliográfica são comentários críticos sobre alguns itens da bibliografia existente que você considera particularmente importantes, seja para neles se apoiar, seja para criticá-los. ${ }^{5}$

A coleta de dados para este estudo foi realizada por meio de artigos científicos publicados de 2008 a 2018. Foram pesquisados os sites da Biblioteca Virtual de Saúde - BVS, Scielo e nas bases de dados LILACS e BDEnf com as palavras chaves: estratégia; conflito; negociação; gestão hospitalar; enfermagem.

Foram encontrados 14 artigos e um livro, somente 10 artigos e um livro, se adequaram para o objetivo desse estudo (Quadro 1). Com eles foi construída uma planilha Excel com os dados para construir essa revisão bibliográfica. Após seleção dos artigos, os mesmos foram lidos, fichados e resultaram em categorias temáticas que compuseram a revisão deste artigo.

Quadro 1. Artigos e livro selecionados.

\begin{tabular}{|l|l|l|l|l|}
\hline No & Título & Autores & Ano & Temática \\
\hline 1 & $\begin{array}{l}\text { O poder de } \\
\text { negociação: Reflexão } \\
\text { sobre o gerenciamento } \\
\text { de conflitos na } \\
\text { enfermagem. }\end{array}$ & $\begin{array}{l}\text { Álvaro Pereira, } \\
\text { Adriana Cristian } \\
\text { Mota, Rudval Souza } \\
\text { da Silva. }\end{array}$ & 2009 & $\begin{array}{l}\text { Identificar as } \\
\text { competências e } \\
\text { habilidades do enfermeiro } \\
\text { no gerenciamento de } \\
\text { conflitos na enfermagem. }\end{array}$ \\
\hline 2 & $\begin{array}{l}\text { Conflitos gerenciais e } \\
\text { estratégias de } \\
\text { resolução pelos } \\
\text { enfermeiros gerentes. }\end{array}$ & $\begin{array}{l}\text { Suzinara Beatriz de } \\
\text { Lima, Liange Arrua } \\
\text { Rabenschlang, } \\
\text { Tanise Finamor } \\
\text { Ferreira, Francislene } \\
\text { Lopes, Ariele do } \\
\text { Nascimento Lampert. }\end{array}$ & 2014 & $\begin{array}{l}\text { Principais estratégias } \\
\text { gerenciais e apontar as } \\
\text { dificuldades encontradas } \\
\text { diante a mediação dos } \\
\text { conflitos no ambiente de } \\
\text { trabalho. }\end{array}$ \\
\hline 3 & $\begin{array}{l}\text { O conflito no exercício } \\
\text { gerencial do } \\
\text { enfermeiro no âmbito } \\
\text { hospitalar. }\end{array}$ & $\begin{array}{l}\text { Soeli Teresinha } \\
\text { Guerra, Adelina } \\
\text { Giacomelli, Maria } \\
\text { Auxiliadora Trevizan, } \\
\text { Laura de Azevedo } \\
\text { Guido. }\end{array}$ & 2011 & $\begin{array}{l}\text { Verificar como os } \\
\text { conflitos se manifestam } \\
\text { nas relações } \\
\text { interpessoais e a } \\
\text { importância que } \\
\text { assumem no exercício do } \\
\text { enfermeiro gerente em }\end{array}$ \\
\hline
\end{tabular}




\begin{tabular}{|c|c|c|c|c|}
\hline & & & & hospitais. \\
\hline 4 & $\begin{array}{l}\text { LIVRO: Competências } \\
\text { gerenciais: Desafio } \\
\text { para o enfermeiro. }\end{array}$ & $\begin{array}{l}\text { Alexandre Pazetto } \\
\text { Balsanelli, Isabel } \\
\text { Cristina Kowal Olm } \\
\text { Cunha, Liliane Bauer } \\
\text { Feldman, Rosa } \\
\text { Maria Ruthes. }\end{array}$ & 2011 & $\begin{array}{l}\text { Apontar as principais } \\
\text { competências de um } \\
\text { enfermeiro gerente, } \\
\text { incluindo as estratégias } \\
\text { mais utilizadas para } \\
\text { resolução de conflitos. }\end{array}$ \\
\hline 5 & $\begin{array}{l}\text { A revisão bibliográfica. } \\
\text { Uma dimensão } \\
\text { fundamental para o } \\
\text { planejamento da } \\
\text { pesquisa. }\end{array}$ & $\begin{array}{l}\text { José D' Assunção } \\
\text { Barros }\end{array}$ & 2009 & $\begin{array}{l}\text { Descrever o que é uma } \\
\text { revisão bibliográfica, } \\
\text { quando e como ela pode } \\
\text { ser usada. }\end{array}$ \\
\hline 6 & $\begin{array}{l}\text { Conflitos gerenciais: } \\
\text { dificuldades para o } \\
\text { enfermeiro gerente. }\end{array}$ & $\begin{array}{l}\text { Ariele do Nascimento } \\
\text { Lampert, Daniella }\end{array}$ & 2013 & $\begin{array}{l}\text { Mostra como os } \\
\text { enfermeiros estão lidando } \\
\text { com conflitos gerenciais a } \\
\text { fim de garantir a } \\
\text { qualidade da assistência } \\
\text { prestada. }\end{array}$ \\
\hline 7 & $\begin{array}{l}\text { A gerência de conflitos } \\
\text { em enfermagem }\end{array}$ & $\begin{array}{l}\text { Rosangela Maria } \\
\text { Greco. }\end{array}$ & 2011 & $\begin{array}{l}\text { Compreender a } \\
\text { importância de gerenciar } \\
\text { um conflito e discutir as } \\
\text { estratégias que facilitam o } \\
\text { gerenciamento de } \\
\text { conflitos na enfermagem. }\end{array}$ \\
\hline 8 & $\begin{array}{l}\text { Conceitos de } \\
\text { estratégia e gestão } \\
\text { estratégica: Qual é o } \\
\text { nível de conhecimento } \\
\text { adquirido pelos } \\
\text { estudantes de gestão? }\end{array}$ & $\begin{array}{l}\text { Emerson Wagner } \\
\text { Mainardes, João } \\
\text { Ferreira, Mário } \\
\text { Raposo. }\end{array}$ & 2010 & $\begin{array}{l}\text { Um estudo para conhecer } \\
\text { o entendimento de alunos } \\
\text { da área de gestão quanto } \\
\text { aos conceitos de } \\
\text { estratégia e gestão } \\
\text { estratégica. }\end{array}$ \\
\hline 9 & $\begin{array}{l}\text { Conflitos éticos da } \\
\text { enfermagem na } \\
\text { atenção primaria á } \\
\text { saúde e estratégias de } \\
\text { enfrentamento. }\end{array}$ & $\begin{array}{l}\text { Przenyczka, Ramone } \\
\text { Aparecida } \\
\text { Kalinowiski, Luísa } \\
\text { Canestraro, Lacerda, } \\
\text { Maria Ribeiro Wall, } \\
\text { Marilene Loewen }\end{array}$ & 2011 & $\begin{array}{l}\text { Identificar conflitos éticos } \\
\text { vivenciados por } \\
\text { enfermeiros em uma } \\
\text { Unidade de Saúde Básica } \\
\text { com Estratégia Saúde da } \\
\text { Família e as estratégias } \\
\text { de enfrentamento mais } \\
\text { utilizada. }\end{array}$ \\
\hline 10 & $\begin{array}{l}\text { Administração de } \\
\text { conflitos: análise de } \\
\text { percepções de } \\
\text { enfermeiros gerentes }\end{array}$ & $\begin{array}{l}\text { Silva, George } \\
\text { Sobrinho, Diniz, } \\
\text { Bruno Gabriel, Rosa, } \\
\text { Vânia Das Graça }\end{array}$ & 2012 & $\begin{array}{l}\text { Analisar as percepções } \\
\text { de enfermeiros em cargos } \\
\text { de gerência em uma } \\
\text { Unidade Básica de } \\
\text { Saúde, apontar as causas } \\
\text { dos conflitos e a atuação } \\
\text { dos gerentes, as } \\
\text { estratégias utilizadas e os } \\
\text { obstáculos encontrados. }\end{array}$ \\
\hline
\end{tabular}




\section{RESULTADOS E DISCUSSÃO}

\section{Conflitos e os aspectos na área da saúde e enfermagem}

Conflitos são resultados de um processo de divergências ou confronto entre indivíduos, organizações ou situações que geram estresse para a equipe mesmo que nem todos estejam envolvidos e precisam ser solucionadas. ${ }^{6}$

Contextualizando com a enfermagem os conflitos estão sempre presentes, o enfermeiro como gestor da unidade e líder de uma equipe precisa ser hábil e estar preparado para resolver os problemas do seu setor. Os conflitos podem ser internos, quando os colaboradores têm problemas pessoais, íntimos de cunho particular ou problemas externos ou sociais, entre eles. ${ }^{7}$

Existem estágios para que se forme um conflito: 1) Conflito latente, onde as divergências são amadurecidas porem internamente e podem vir ou não a acontecer; 2) Conflito percebido, quando está em evolução; 3) Conflito sentido, quando as emoções vêm à tona, tais como raiva, hostilidade, desconfiança ou até medo; 4) Conflito manifesto, quando o sentimento é exteriorizado e atinge ambas as partes. ${ }^{3}$

Como em qualquer lugar em que se tenham pessoas trabalhando em conjunto haverá conflitos, na enfermagem não é diferente, são equipes grandes, cada um com suas prioridades e individualidades, trabalhando muitas vezes com falta de funcionários, com escalas de trabalho apertadas, com falta de recursos humanos e materiais, entre outros problemas que podem surgir. $\mathrm{O}$ conflito em si não é uma situação negativa, com ele vão surgindo mudanças que podem melhorar as rotinas quando resolvido, passa a ser um problema quando se torna um confronto. ${ }^{7}$

Os conflitos são inerentes a vida das pessoas, e podem ser considerados como situações de discordâncias sejam elas internas ou externas, como resultado de ideias, valores ou sentimentos diferentes. Assim quando pensamos em uma empresa, instituição ou serviço é natural que por serem constituídas por pessoas com histórias de vida diferentes, conceitos, valores, uma série de aspectos que fazem com que cada um seja um ser único e insubstituível, é normal e até esperado que os conflitos se façam presentes. ${ }^{7}$

As principais causas dos conflitos identificados estariam relacionadas à falta de empatia, divergências de opiniões, dificuldade de se relacionar, problemas particulares, duplicidade de vínculo empregatício, insegurança, falta de respeito pelo trabalho do outro, escassez de recursos humano e material. ${ }^{2}$

O conflito é como resultado de disputa de interesses de cunho social e que invadem a organização. Disputa de papéis em nível organizacional, relacionado à crescente necessidade de controle dos gerentes sobre a coletividade de trabalhadores. Conflito como fruto das diferentes personalidades, percepções, falta de comunicação, estrutura organizacional, escalas de serviço 
sobrecarregado, mal-entendidos, falta de compromisso, falta de cooperação, desmotivação e desvalorização no trabalho. ${ }^{6}$

Grande parte dos enfermeiros gerentes mostra-se ciente de que a resolução de conflitos é uma atividade inerente ao cargo, é indispensável para o êxito da organização e embora seja uma atividade corriqueira, não é vista como uma tarefa simples de lidar, por sempre envolver indivíduos "tensos" e "interesses distintos". 10

Os conflitos interpessoais costumam interferir no andamento do trabalho e por consequência afetam o desempenho destes trabalhadores, de certo modo implicando na qualidade da assistência aos usuários. ${ }^{4}$

\section{Estratégias de negociação na enfermagem}

O termo estratégia origina-se do grego "strategos", sendo traduzida como "general no comando de tropas". A estratégia já era considerada habilidade administrativa na época de Péricles (450 a.C.), significando habilidades gerenciais (administrativas, liderança, oratória, poder). Mas foi somente depois da Segunda Guerra Mundial que a estratégia passou a fazer parte da vida das empresas, que cresceram significativamente e precisavam de diretrizes, linhas e caminhos a serem seguidos por toda a sua estrutura. ${ }^{8}$

Atualmente o mercado de trabalho busca um perfil de enfermeiro mais dinâmico, qualificado, empreendedor, com bom desenvolvimento em equipe, bem informado, que seja flexível, comprometido, e que principalmente visualize a frente propondo melhorar e inovar. A criatividade é considerada fundamental para o enfermeiro. ${ }^{4}$

O enfermeiro assume o papel de suma importância, para que os enfermeiros possam desenvolver uma visão estratégica; é preciso designar um tempo para ouvir o seu maior patrimônio, no caso os enfermeiros que atuam nas diversas unidades. É importante ressaltar também que o gerente é um educador, e como tal deverá desempenhar uma liderança inovadora, estimulando a sua equipe a pensar e desenvolvendo objetivos e resultados. A inovação na enfermagem de qualquer instituição pode estar na ideia de uma dessas pessoas, então, é necessário um tempo para escutá-las, para conhecer suas visões, suas necessidades, o perfil delas como equipe, sua forma e seu campo de atuação. Nesse simples comportamento estratégico, pode estar a resposta para a mudança. ${ }^{4}$

Nesse sentido, reuniões para discussão de casos e feedback, são essenciais para detecção de problemas, diminuindo e identificando precocemente adversidades que podem ser evitadas. ${ }^{9}$

A negociação é um processo no qual existem duas partes que passam por uma transação, em que são empregadas estratégias para resolução de conflitos, com a finalidade de chegar a um acordo mútuo. Dentre os vários momentos existentes durante a negociação destacam-se algumas etapas a serem 
cumpridas: planejamento; quebra de gelo; exploração; confronto. Confiança, persuasão, conhecimento lógico, adaptabilidade, entre outros, são apenas alguns fatores básicos para o negociador. ${ }^{4}$

O uso da negociação, através de um diálogo aberto entre os envolvidos sempre foi apontado como a principal alternativa para se buscar um consenso em ambas às partes, porém a forma de se conduzir uma negociação se diferencia de acordo com os personagens. Há a preferência de uso do estilo colaborador, buscando soluções pelos quais os dois lados se mantenham preservados. A necessidade de recorrer às relações de poder, especificamente à hierarquia para resolução de conflitos, na maioria das vezes é identificada como falha no processo de negociação e a necessidade de resolver um impasse. ${ }^{10}$

O ideal é que todos estejam engajados no mesmo objetivo, para alcançar os melhores resultados e sempre utilizando a melhor estratégia. ${ }^{4}$

Antes da escolha da melhor estratégia, é preciso considerar um conjunto de fatores, tais como as particularidades de cada situação, a urgência da tomada de decisão a importância da questão em foco. Dessa forma cabe ao profissional, conhecer a realidade na qual está inserido: tipo de instituição, missão social, equipe, entre outros. ${ }^{4}$

Hoje em dia se dispõe de muitas estratégias para resolver conflitos, desde a confrontação, compromisso, abrandamento, competição, colaboração, negociação, evitar conflito ou reprimi-lo, transformar a diferença em resolução de problema, mudanças comportamentais, conhecimento, habilidades e atitudes. ${ }^{10}$

As estratégias podem ser apreciadas na Figura 1. 


\section{ESTRATÉGIAS RESOLUTIVAS}

\section{COMUNICAÇÃO EFETIVA E FEEDBACK}

A comunicação é de extrema importância entre o enfermeiro e seus colaboradores pois facilita 0 relacionamento interpessoal e desenvoltura do trabalho em equipe.

0 feedback é necessário para avaliação de ações ou resultados obtidos individualmente ou em grupo.

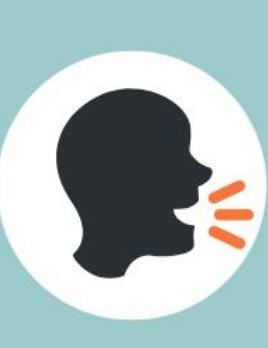

\section{CAPACIDADE DE OBSERVAÇÃO}

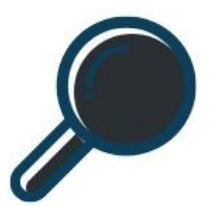

Apesar de tantas funções a serem realizadas, o Enfermeiro precisa ter uma visão holística, estar atento à sua equipe, às rotinas do setor, evitando perda de qualidade no atendimento e auxiliando no crescimento dos seus funcionários

\section{DISCERNIMENTO}

Envolve competências necessárias, habilidades como sensatez e clareza para compreender situações, discrição e agir sempre com ética.

\section{COMPROMETIMENTO}

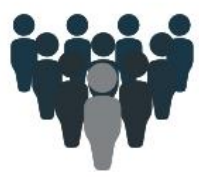

Estar em conjunto com sua equipe, definindo acordos que sejam bons para todos os envolvidos e respeitando - os sejam eles escritos ou verbais, formais ou informais.

\section{LIDERANÇA}

Ter postura de líder, sendo responsável. Conduzindo sua equipe, motivando para alcançar melhores resultados, valorizando habilidades, respeitando dificuldades, saber distribuir tarefas para que não haja sobrecarga e manter um ambiente de trabalho saudável.

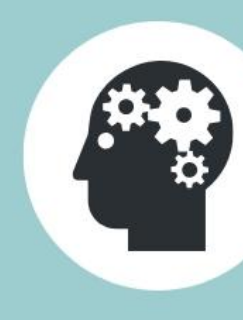

Figura 1. Estratégias resolutivas - Infográfico 
No infográfico criado (Figura 1), foram apontados conhecimentos, habilidades e atitudes (CHA) necessárias para os enfermeiros realizarem suas atividades administrativas. CHA é um conjunto de competências estratégicas essenciais que o enfermeiro deve ter para utilizar em sua gestão, para evitar, lidar e solucionar conflitos. Possuir estas competências como estratégias é o diferencial do profissional, dessa forma é importante que o mesmo saiba onde está inserido, tipo de instituição, valores, pontos fortes e serviços a serem prestados.

\section{CONSIDERAÇÕES FINAIS}

A partir desse estudo, pode-se analisar as estratégias mais utilizadas pelo enfermeiro para resolução de conflitos, destacando temas de supra importância para a compreensão do assunto.

Percebe-se que conflitos surgem durante a interação interpessoal cotidiana do trabalho e seu desencadeamento está relacionado à forma como cada um conduz e responde às interações.

Assim, as principais causas dos conflitos encontradas estariam relacionadas à falta de empatia, divergências de opiniões, dificuldade de se relacionar e a problemas particulares, lidar com certos conflitos nem sempre será fácil e a negociação é uma ferramenta fundamental para o término do processo, onde ambas as partes possam entrar em um acordo.

Gerenciar pessoas, dentre todas as variáveis administrativas, é apontada como a mais difícil e importante para o êxito da organização, com destaque para o manejo dos conflitos e as situações envolvidas.

É primordial analisar as situações, respeitar as diferenças tanto socioeconômicas, como culturais de cada pessoa, ter um bom diálogo, ser bom ouvinte e se posicionar perante sua equipe criando um vínculo, fazendo com que se sintam à vontade para levar uma situação à gerência, ganhando espaço como líder e a confiança de seus colaboradores. No entanto, para que isso aconteça, o enfermeiro precisa conhecer a sua equipe, a instituição onde atua, missão, entre outros. Vale ressaltar que o enfermeiro no seu papel de líder deverá possuir conhecimento, habilidades e atitudes, buscando sempre uma liderança inovadora e criativa. Conquistando um ambiente de trabalho mais harmonioso e prazeroso.

Portanto buscou-se identificar quais as estratégias utilizadas na resolução de conflitos pelo enfermeiro gestor através dos principais conflitos achados.

\section{REFERÊNCIAS}

1. Pereira, Álvaro; Lima, Adriana Cristian Mota Venas; Silva, Rudval Souza da. O poder da negociação: reflexão sobre o gerenciamento de conflitos na enfermagem. Rev Enf Online 2009 Jan/Mar; 3(1): 117-23 ISSN: 1981-8963. 
2. Lima, Suzinara Beatriz Soares de; Rabenschlag, Liange Arrua; Tonini, Tanise Finamor Ferreira; Menezes, Franscilene Lopes; Lampert, Ariele do Nascimento. Conflitos gerenciais e estratégias de resolução pelos enfermeiros gerentes. Rev Enf UFSM 2014 Abr/Jun; 4(2): 419-428 ISSN: 2179-7692.

3. Guerra, Soeli Teresinha; Prochnow, Adelina Giacomelli; Trevizan, Maria Auxiliadora; Guido, Laura de Azevedo. O conflito no exercício gerencial do enfermeiro no âmbito hospitalar. Rev Latino-Am Enf 2011 Mar/Abr; 19(2).

4. Balsanelli, Alexandre Pazetto; Cunha, Isabel Cristina Kowal; Feldman, Liliane Bauer; Ruthes, Rosa Maria. Competências gerencias: desafio para o enfermeiro. $2^{\circ}$ ed. São Paulo: Martinari; 2011.

5. Barros, José D' Assunção. A Revisão Bibliográfica: uma dimensão fundamental para o planejamento de pesquisa. Instr Rev Est Pesq Educ Juiz de Fora, 2009 jul./dez; 1(2): 103-111,

6. Lampert, Ariele do Nascimento; Kinalski, Daniella Dal Forno; Machado, Bruna Parvo; Lima, Beatriz Soares de. Conflitos gerenciais: dificuldades para enfermeiro gerente. REAS [Internet] 2013; 2(3): 96-105 ISSN: 23171154.

7. Greco, Rosangela Maria. A gerência de conflitos em enfermagem. Ufjf Material instrucional para Administração em enfermagem II, Juiz de Fora: Departamento de enfermagem básica; 2011.

8. Mainardes, Emerson Wagner; Ferreira, João; Raposo. Conceitos de estratégia e gestão estratégica: qual é o nível de conhecimento adquirido pelos estudantes de gestão?. FACEF Pesq Fraca 2011 Set/Out/ Nov/Dez; 14(3): 278-298 ISSN: 1516-6503.

9. Przenyczka, Ramone Aparecida; Kalinowski, Luísa Canestraro; Lacerda, Maria Ribeiro; Wall, Marilene Loewen. Conflitos éticos da enfermagem na atenção primaria à saúde e estratégias de enfrentamento. Cienc Cuid Saude 2011 Abr/Jun; 10(2): 330-337

10. Silva, George Sobrinho; Diniz, Bruno Gabriel; Rosa, Vânia das Graças. Administração de conflitos: análise de percepções de enfermeiros gerentes. Revi Enferm Cent O Min 2012 Set/Dez; 2(3): 358-368.

Recebido: 23 de novembro de 2018. Publicado: 28 de dezembro de 2018

Correspondência: Ana Caroline Duarte de Sousa. E-mail: carolinee_dds@hotmail.com 
Conflito de Interesses: os autores declararam não haver conflito de interesses.

(C) This is an Open Access article distributed under the terms of the Creative Commons Attribution License, which permits unrestricted use, distribution, and reproduction in any medium, provided the original work is properly cited 